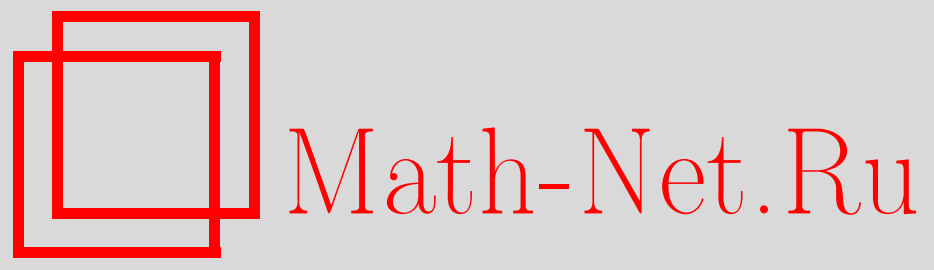

А. Н. Фролов, Предельные теоремы для приращений сумм независимых случайных величин, Теория вероятн. и ее примен., 2003, том 48, выпуск 1, 104121

DOI: https://doi.org/10.4213/tvp303

Использование Общероссийского математического портала MathNet.Ru подразумевает, что вы прочитали и согласны с пользовательским соглашением

http://www . mathnet.ru/rus/agreement

Параметры загрузки:

IP: 54.224 .60 .19

26 апреля 2023 г., $12: 32: 34$

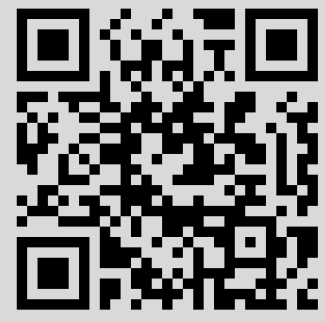




\section{ПРЕДЕЛЬНЫЕ ТЕОРЕМЫ ДЛЯ ПРИРАЩЕНИЙ СУММ НЕЗАВИСИМЫХ СЛУЧАЙНЫХ ВЕЛИЧИН ${ }^{1)}$}

Исследовано асимптотическое поведение почти наверное приращений сумм независимых одинаково распределенных случайных величин, удовлетворяющих одностороннему условию Крамера. Установлено, что нормирующая последовательность в сильных предельных теоремах для приращений сумм, независимо от их длины, определяется поведением функции, обратной к функции уклонений. Это позволяет объединить единой формулировкой следующие известные результаты для приращений сумм: закон больших чисел, закон Эрдёша-Реньи и его расширение, полученное Мэйсоном, закон Шеппа, теоремы Чёргё-Ревеса, закон повторного логарифма. В случае больших прирашений получены новые результаты для случайных величин из области притяжения устойчивого закона с характеристическим показателем $\alpha \in(1,2]$ и параметром симметрии $\beta=-1$.

Ключевые слова и фразы: прирашения сумм независимых случайных величин, большие уклонения, законы Эрдёша-Реньи и Шеппа, законы сильной аппроксимации, закон больших чисел, закон повторного логарифма.

1. Введение и основные результаты. Пусть $X, X_{1}, X_{2}, \ldots$ последовательность независимых одинаково распределенных случайных величин, $S_{n}=X_{1}+X_{2}+\cdots+X_{n}, S_{0}=0,\left\{a_{n}\right\}$ - неубывающая последовательность натуральных чисел, $1 \leqslant a_{n} \leqslant n$.

Исследование асимптотического поведения почти наверное максимуMOB

$$
U_{n}=\max _{0 \leqslant k \leqslant n-a_{n}}\left(S_{k+a_{n}}-S_{k}\right)
$$

описывающих, например, колебания максимального выигрыша игрока в $a_{n}$ последовательных партиях некоторой игры, представляет существенный интерес. (Отметим, что $U_{n}=S_{n}$ при $a_{n}=n$ и $U_{n}=$

${ }^{*}$ С.-Петербургский государственный университет; Старый Петергоф, Университетский пр. 28, 198504 С.-Петербург, Россия; e-mail: andrei.frolov@pobox.spbu.ru

1) Работа выполнена при поддержке Российского фонда фундаментальных исследований, грант № 99-01-00732, и гранта ведущих научных школ № 00-15-96019. 
$\max _{1 \leqslant k \leqslant n} X_{k}$ при $a_{n}=1$.) Имеющиеся в этом направлении работы посвящены отысканию условий, необходимых и/или достаточных для того, чтобы

$$
\lim \sup \frac{U_{n}}{b_{n}}=1 \text { п.н., }
$$

где $\left\{b_{n}\right\}$ - некоторая последовательность положительных постоянных. (Всюду, где использованы символы $\lim \sup , \lim \inf , \lim , O, o, \rightarrow$, считаем, что $n \rightarrow \infty$, если не оговорено противное.) В зависимости от скорости роста $a_{n}$ все полученные ранее результаты делятся на два типа. Если $a_{n}=O(\ln n)$, то нормирующая последовательность $\left\{b_{n}\right\}$ существенно зависит от распределения слагаемых, а иногда даже и определяет это распределение. Этому случаю посвящены работы [1]-[5]. Если $a_{n} / \ln n \rightarrow \infty$, то нормируюшая последовательность универсальна и совпадает с нормировкой для гауссовского распределения. Этот случай рассматривался в работах [6]-[8]. Все эти результаты можно охватить единой формулировкой, в которую оказываются включенными следующие известные сильные предельные теоремы для приращений сумм: закон больших чисел, закон Эрдёша-Реньи и его расширение, полученное Мэйсоном, закон Шеппа, закон Чёргё-Ревеса, закон повторного логарифма.

Наряду с $U_{n}$ мы рассмотрим

$$
W_{n}=\max _{0 \leqslant k \leqslant n-a_{n}} \max _{1 \leqslant j \leqslant a_{n}}\left(S_{k+j}-S_{k}\right), \quad R_{n}=S_{n}-S_{n-a_{n}}, \quad T_{n}=S_{n+a_{n}}-S_{n} .
$$

Отметим, что $W_{n}=\max _{1 \leqslant k \leqslant n} S_{k}$ при $a_{n}=n$ и $W_{n}=\max _{1 \leqslant k \leqslant n} X_{k}$ при $a_{n}=1$.

Предположим, что выполнены следующие условия:

(А) случайная величина $X$ невырождена;

(B) $0 \leqslant \mathbf{E} X<\infty$;

(C) $h_{0}=\sup \left\{h: \varphi(h)=\mathbf{E} e^{h X}<\infty\right\}>0$.

Обозначим $F(x)=\mathbf{P}\{X<x\}, \omega=\operatorname{ess} \sup X$. Положим для $0<h<h_{0}$

$$
m(h)=\frac{\varphi^{\prime}(h)}{\varphi(h)}, \quad \sigma^{2}(h)=m^{\prime}(h), \quad f(h)=h m(h)-\ln \varphi(h) .
$$

Обозначим $A=\lim _{h \succ h_{0}} m(h), 1 / c_{0}=\lim _{h \succ h_{0}} f(h)$,

$$
\zeta(z)=\sup \{z h-\ln \varphi(h): h \geqslant 0, \varphi(h)<\infty\}, \quad \gamma(x)=\sup \{z: \zeta(z) \leqslant x\} .
$$

Свойства функций $m(h), \sigma^{2}(h)$ и $\zeta(z)$ хорошо известны из теории больших уклонений (см., например, [9], [10]). Функции $m(h)$ и $\sigma^{2}(h)$ - это соответственно математическое ожидание и дисперсия преобразования Крамера случайной величины $X$, а $\zeta(z)$ - функция уклонений. Функции 
$m(h), f(h), \zeta(z)$ и $\gamma(x)$ связаны следуюшими соотношениями:

$$
\begin{array}{lll}
\zeta(z)=f\left(m^{-1}(z)\right) & \text { при } & z \in[\mathbf{E} X, A), \\
\gamma(x)=m\left(f^{-1}(x)\right) & \text { при } & x \in\left[0, \frac{1}{c_{0}}\right),
\end{array}
$$

где $m^{-1}(z)$ и $f^{-1}(x)$ - это функции, обратные к $m(h)$ и $f(h)$ соответственно. Функция $\gamma(x)$ обладает следующими свойствами: $\gamma(0)=\mathbf{E} X$, $\gamma(x) \rightarrow \omega$ при $x \rightarrow \infty, \gamma(x)$ выпукла вверх.

В зависимости от того, каковы постоянные $h_{0}, A$ и $c_{0}$, существует пять классов распределений:

I. $h_{0}=\infty, A<\infty, c_{0}=0$. В этом случае $\omega=A$.

II. $h_{0}=\infty, A<\infty, c_{0}>0$. В этом случае $\omega=A, c_{0}=$ $-1 / \ln \mathbf{P}\{X=A\}$.

III. $h_{0}=\infty, A=\infty$. В этом случае $\omega=\infty, c_{0}=0$.

IV. $h_{0}<\infty, A=\infty$. В этом случае $\omega=\infty, c_{0}=0$.

V. $h_{0}<\infty, A<\infty$. В этом случае $\omega=\infty, c_{0}=\left(h_{0} A-\ln \varphi\left(h_{0}\right)\right)^{-1}>0$. (См. также [9] и [4].)

Например, классам I-V принадлежат соответствено равномерное, бернуллиевское, нормальное, экспоненциальное распределения и распределение с плотностью $p(x)=C x^{-3} e^{-x}, x \geqslant 1$.

Далее запись $F \in K_{i}$ обозначает, что распределение $F$ принадлежит классу $i, i=\mathrm{I}, \ldots, \mathrm{V}$.

Для $F \in K_{\mathrm{V}}$ доопределим функции $m(h)$ и $f(h)$ для $h \geqslant h_{0}$ следующим образом:

$$
m(h)=m\left(h_{0}\right)+h-h_{0}, \quad f(h)=f\left(h_{0}\right)+h_{0}\left(h-h_{0}\right) .
$$

В этом случае соотношение (1) будет выполнено для всех $z \geqslant \mathbf{E} X$, a (2)для всех $x \geqslant 0$.

Предположим, что $a_{n} / a(n) \rightarrow 1$, где $a(x)$ - неубывающая функция такая, что $1 \leqslant a(x) \leqslant x, x / a(x)$ не убывает. Пусть функция

$$
b(x)=a(x) \gamma\left(\frac{1}{a(x)}\left(\ln \frac{x}{a(x)}+\ln \ln x\right)\right)
$$

не убывает. Положим

$$
b_{n}=a_{n} \gamma\left(\frac{1}{a_{n}}\left(\ln \frac{n}{a_{n}}+\ln \ln n\right)\right) .
$$

Всюду в дальнейшем $[x]$ обозначает целую часть $x$.

Сформулируем сначала результат, дающий представление о наибольшей возможной скорости роста максимальных прирашений сумм независимых случайных величин. 
Теорема 1. Пусть выполнены условия (A), (В) $u$ (C). Пусть сушествует функчия $g(\theta), \theta \in\left(1, \theta_{0}\right)$, такая, ито $g(\theta) \rightarrow 1$ при $\theta \rightarrow 1 u$ $b(\theta x) / b(x) \leqslant g(\theta)$ при всех достаточно больших $x$.

Предположим, что выполнено одно из следующих условий:

1) $\ln a_{n} / \ln n \rightarrow 0$

2) для любого $\varepsilon>0$ существует $q \in(0,1)$ такое, что для всех $i \leqslant I_{n}=\min \left\{\left[(1+\varepsilon) a_{n}\right], n\right\}$ и всех достаточно больших $n$ выполняется неравенство

$$
\mathbf{P}\left\{S_{i} \geqslant-\varepsilon b_{n}\right\} \geqslant q
$$

Tогдa

$$
\limsup \frac{W_{n}}{b_{n}} \leqslant 1 \quad \text { n.н. }
$$

3 а м е ч а н и е. Если $\left|I_{n+1}-I_{n}\right| \leqslant C$ для всех достаточно больших $n$ и $b_{n} \rightarrow \infty$, то неравенство (4) достаточно проверить только для $i=I_{n}$. В частности, если $a_{n}=[a(n)]$, то $\left|I_{n+1}-I_{n}\right| \leqslant 3$. Условие $b_{n} \rightarrow \infty$ может не выполняться только для распределений из классов I и II. Для таких распределений при $a_{n}=o(\ln n)$ заключение теоремы 1 выполнено автоматически, так как в этом случае $\omega<\infty$ и $b_{n} \sim a_{n} \omega$.

Перейдем к оценкам снизу для скорости роста приращения сумм независимых случайных величин.

Теорема 2. Пусть выполнены условия (A), (B) $u(\mathrm{C})$. Для $\varepsilon \in(0,1)$ положим

$$
h^{*}=f^{-1}\left(\frac{1-\varepsilon}{a_{n}}\left(\ln \frac{n}{a_{n}}+\ln \ln n\right)\right) .
$$

Предположим, что выполнено одно из следующих условий:

1) для всех достаточно мальх $\varepsilon \in(0,1)$ справедливы неравенство $h^{*}<h_{0}$ и условие

$$
h^{*} \sigma\left(h^{*}\right)=o\left(\sqrt{a_{n}} f\left(h^{*}\right)\right)
$$

2) $F \in K_{\mathrm{I}} u h^{*} \rightarrow \infty$;

3) $F \in K_{\mathrm{II}} u\left(\ln \left(n / a_{n}\right)+\ln \ln n\right) / a_{n} \geqslant 1 / c_{0}$;

4) $F \in K_{\text {III }}$ uлu $F \in K_{\mathrm{V}}, h^{*} \rightarrow \infty u$

$$
\mathbf{P}\{X \geqslant(1-\tau) m(h)\} \geqslant \exp \{-(1+\delta) f(h)\}
$$

для любых $\tau>0, \delta>0$ и всех достаточно больших $h$;

5) $F \in K_{\mathrm{IV}}, h^{*} \nearrow h_{0}$ и неравенство (6) выполнено для любых $\tau>0$, $\delta>0$ ивсех $h$, достаточно близких $\kappa h_{0}$;

6) $F \in K_{\mathrm{V}}, h^{*}>h_{0}, h^{*}$ не зависит от $n$.

Если $a_{n} / n \rightarrow 1$, то предположим дополнительно, что выполнено условие 2) теоремь 1 . 
Тогда

$$
\lim \sup \frac{R_{n}}{b_{n}} \geqslant 1 \text { n.н. }
$$

$B$ последнем соотношении $R_{n}$ можно заменить на $T_{n}$.

Условия 1)-6) теоремы 2 позволяют охватить практически весь возможный диапазон последовательностей $\left\{a_{n}\right\}$ для всех пяти классов распределений.

Если $F \in K_{\mathrm{I}}$, то условие 1) нужно проверять для $h^{*}=O(1)$ $\left(\liminf a_{n} / \ln n>0\right)$. Условие 2) следует использовать, если $h^{*} \rightarrow \infty$ $\left(a_{n}=o(\ln n)\right)$.

Если $F \in K_{\text {II }}$, то областью значений функции $f(h)$ является интервал $\left[0,1 / c_{0}\right)$. Поэтому условие 1$)$ можно применять только для $a_{n}>c_{0} \ln n$. Случай $a_{n} \leqslant c_{0} \ln n$ включен в 3$)$.

Если $F \in K_{\text {III }}$ или $F \in K_{\text {IV }}$, то условие 1$)$ удобно проверять для $h^{*}=$ $O(1)\left(\lim \inf a_{n} / \ln n>0\right)$. Условия 4) и 5) могут быть проше условия 1) при $h^{*} \rightarrow h_{0}\left(a_{n}=o(\ln n)\right)$.

Если $F \in K_{\mathrm{V}}$, то условие 1) удобно использовать для $h^{*}=O(1)$ $\left(\liminf a_{n} / \ln n>0\right)$, условие 6$)$ следует применять для фиксированного $h^{*}>h_{0}\left(a_{n}=c \ln n, 0<c<c_{0}\right)$, а условие 4) нужно проверять для $h^{*} \rightarrow \infty\left(a_{n}=o(\ln n)\right)$.

Отметим, что в некоторых случаях условие 1) и дополняющее его условие могут выполняться одновременно.

Условия 1)-6) теоремы 2 можно заменить любым условием, позволяющим получить следующую оценку снизу вероятностей больших уклонений:

$$
\mathbf{P}\left\{S_{n} \geqslant(1-\varepsilon) n m\left(h_{n}\right)\right\} \geqslant \exp \left\{-n f\left(h_{n}\right)(1+\delta)\right\}
$$

для любых $\varepsilon, \delta>0$ и всех достаточно больших $n$.

Отметим, что если выполнено одно из условий 2)-6), то мы приходим к результатам из [4] и [5]. Однако в случаях 3) и 6) доказательства в [4] нуждаются в некоторых дополнениях и изменениях. Заметим, что наши доказательства универсальны.

Наш следующий результат содержит оценку снизу скорости роста максимальных приращений сумм независимых случайных величин для достаточно медленно возрастающих последовательностей $\left\{a_{n}\right\}$.

Теорема 3. Если выполнены условия теоремы $2 u \ln \ln n=$ $o\left(\ln \left(n / a_{n}\right)\right), m o$

$$
\liminf \frac{U_{n}}{b_{n}} \geqslant 1 \quad \text { n.н. }
$$

Из теорем 1-3 и неравенств $R_{n} \leqslant U_{n} \leqslant W_{n}$ вытекает следуюшая теорема, дающая обшее представление о поведении почти наверное приращений сумм независимых случайных величин. 
Теорема 4. Если выполнены условия теорем 1 и 2, то

$\lim \sup \frac{W_{n}}{b_{n}}=\limsup \frac{U_{n}}{b_{n}}=\lim \sup \frac{R_{n}}{b_{n}}=\lim \sup \frac{T_{n}}{b_{n}}=1 \quad$ n.н.

Если дополнительно $\ln \ln n=o\left(\ln \left(n / a_{n}\right)\right)$, то

$$
\lim \frac{W_{n}}{b_{n}}=\lim \frac{U_{n}}{b_{n}}=1 \quad \text { n.н. }
$$

Приведем различные важные следствия из теоремы 4. При этом мы переносим полную проверку соответствующих условий в п 2. Наша ближайшая цель - показать, как в различных случаях находить нормирующую последовательность $\left\{b_{n}\right\}$.

I) Пусть $a_{n}=[c \ln n]$. В этом случае условие (5) выполнено автоматически. Теорема 4 влечет следующее соотношение:

$$
\lim \frac{W_{n}}{a_{n}}=\lim \frac{U_{n}}{a_{n}}=\lim \sup \frac{T_{n}}{a_{n}}=\gamma\left(\frac{1}{c}\right) \text { п.н. }
$$

Это полная форма законов Эрдёша-Реньи и Шеппа, полученная в [1]-[4].

II) Пусть $a_{n}=o(\ln n)$. Мы имеем из (8)

$$
\lim \frac{W_{n}}{a_{n} \gamma\left(\ln n / a_{n}\right)}=\lim \frac{U_{n}}{a_{n} \gamma\left(\ln n / a_{n}\right)}=1 \text { п.н. }
$$

Результат, несколько лучший, чем этот, получен Мэйсоном [5]. Он доказал, что если выполнены условия (A), (B), (C), то

$$
\limsup \frac{U_{n}}{a_{n} \gamma\left(\ln n / a_{n}\right)}=1 \text { п.н. }
$$

В последнем соотношении $\lim \sup$ можно заменить на $\lim$, если $\omega<\infty$ или $\lim _{x \rightarrow \infty} \gamma(-\ln (1-F(x))) / x=1$. Отметим, что последнее условие Мэйсона эквивалентно условию (6). Согласно [5], условие (6) выполнено для нормального, геометрического, пуассоновского распределений и для распределения Вейбулла.

III) Пусть $a_{n} / \ln n \rightarrow \infty$. Рассмотрим отдельно следующие случаи:

1. $\mathbf{E} X=0, \sigma^{2}=\mathbf{E} X^{2}<\infty$. Далее мы покажем, что в этом случае

$$
\begin{aligned}
\varphi(h) & =1+\frac{\sigma^{2} h^{2}}{2}(1+o(1)), \quad m(h)=\sigma^{2} h(1+o(1)), \\
\sigma^{2}(h) & =\sigma^{2}(1+o(1)), \quad f(h)=\frac{\sigma^{2} h^{2}}{2}(1+o(1))
\end{aligned}
$$

при $h \rightarrow 0$. Отсюда следует соотношение (5). Из (9), (10) и (2) получаем, что $\gamma(x) / \sqrt{2 \sigma^{2} x} \rightarrow 1$ при $x \rightarrow 0$. (Отметим, что для нормального распределения $\gamma(x)=\sqrt{2 \sigma^{2} x}$ для всех $x \geqslant 0$.) Поэтому соотношения (7) и (8) теоремы 4 выполнены при

$$
b_{n}=\left(2 \sigma^{2} a_{n}\left(\ln \left(\frac{n}{a_{n}}\right)+\ln \ln n\right)\right)^{1 / 2} .
$$


Мы приходим к обобщению закона Чёргё-Ревеса [6], полученному автором в [8]. Отметим, что автор в [8] показал, что условие (C) является необходимым для того, чтобы $\lim \sup W_{n} / b_{n}=1$ п.н. для всех последовательностей $\left\{a_{n}\right\}$ таких, что $a_{n} / \ln n \rightarrow \infty$.

Если $a_{n}$ удовлетворяет условию $\ln \left(n / a_{n}\right) / \ln \ln n \rightarrow c \geqslant 0$, то мы получаем из (7) следующую форму закона повторного логарифма для приращений:

$$
\limsup \frac{W_{n}}{\sqrt{2 n \ln \ln n}}=\lim \sup \frac{U_{n}}{\sqrt{2 n \ln \ln n}}=\sqrt{1+c} \sigma \quad \text { п.н. }
$$

При $a_{n}=n$ последнее соотношение преврашается в утверждение закона повторного логарифма для $S_{n}$ и $\max _{1 \leqslant k \leqslant n} S_{k}$.

2. $\mathbf{E} X=0, \mathbf{E} X^{2}=\infty$. Здесь мы рассмотрим три случая.

a) Предположим, что $F$ принадлежит области притяжения нормального закона. Мы покажем далее, что в этом случае

$$
\begin{aligned}
\varphi(h) & =1+\frac{h^{2}}{2} G\left(\frac{1}{h}\right)(1+o(1)), & m(h) & =h G\left(\frac{1}{h}\right)(1+o(1)), \\
\sigma^{2}(h) & =G\left(\frac{1}{h}\right)(1+o(1)), & f(h) & =\frac{h^{2}}{2} G\left(\frac{1}{h}\right)(1+o(1))
\end{aligned}
$$

при $h \rightarrow 0$. Здесь $G(x)=\int_{-x}^{0} y^{2} d F(y)$. Условие (5) следует из (11) и (12). Постоянные $b_{n}$, при которых выполняются соотношения (7) и (8), определяются из соотношений $(11),(12)$ и (2).

Этот случай ранее не рассматривался.

b) Предположим, что $F$ - функция распределения устойчивого закона с характеристической функцией

$$
\psi(t)=\exp \left\{-c|t|^{\alpha}\left(1+i \frac{t}{|t|} \operatorname{tg} \frac{\pi}{2} \alpha\right)\right\}, \quad \alpha \in(1,2),
$$

где $c=\cos (\pi(2-\alpha) / 2)$. В этом случае условие (C) выполнено, а $\varphi(h)=$ $\exp \left\{h^{\alpha}\right\}, h>0$ (см. [12, с. 80]). Это дает

$$
m(h)=\alpha h^{\alpha-1}, \quad \sigma^{2}(h)=\alpha(\alpha-1) h^{\alpha-2}, \quad f(h)=(\alpha-1) h^{\alpha}
$$

при $h>0$. Отсюда следуют соотношение (5) и, с учетом (2), равенство $\gamma(x)=\alpha(\alpha-1)^{(1-\alpha) / \alpha} x^{(\alpha-1) / \alpha}$. Поэтому соотношение (7) теоремы 4 выполнено при

$$
b_{n}=\alpha(\alpha-1)^{(1-\alpha) / \alpha} a_{n}^{1 / \alpha}\left(\ln \left(\frac{n}{a_{n}}\right)+\ln \ln n\right)^{(\alpha-1) / \alpha} .
$$

Этот результат фактически был получен в [7], где рассматривалось поведение устойчивого процесса. Наша теорема 4 не только дает соотношение для limsup, но и позволяет заменить $\lim \sup$ на $\lim$ для $a_{n}$, удовлетворяюших условию $\ln \ln n=o\left(\ln \left(n / a_{n}\right)\right)$. 
c) Пусть $F$ принадлежит области притяжения устойчивого закона с характеристической функции $\psi(t)$, определенной в (13). Как мы покажем далее, в этом случае

$$
\begin{aligned}
\varphi(h) & =1+c_{1} h^{\alpha} g\left(h^{-1}\right)(1+o(1)), \quad m(h)=c_{2} h^{\alpha-1} g\left(h^{-1}\right)(1+o(1)), \\
\sigma^{2}(h) & =c_{3} h^{\alpha-2} g\left(h^{-1}\right)(1+o(1)), \quad f(h)=c_{4} h^{\alpha} g\left(h^{-1}\right)(1+o(1))
\end{aligned}
$$

при $h \rightarrow 0$, где $g(x)=x^{\alpha} F(-x), x>0$. Отсюда следует (5). Постоянные $b_{n}$, при которых выполняются соотношения (7) и (8), определяются из (14), (15) и (2).

Если $F$ принадлежит области нормального притяжения устойчивого закона, то нормирующие постоянные $b_{n}$ те же, что и в п. b). Соотношение (7) доказано в [7] при более слабых моментных предположениях для $a_{n}$, удовлетворяющих условию $n^{\alpha} / b_{n} \rightarrow \infty$. Предположение о конечности экспоненциального момента существенно расширяет класс рассматриваемых последовательностей $\left\{a_{n}\right\}$. Кроме того, в силу (8) мы можем заменить $\lim \sup$ на $\lim$ для $a_{n}$, возрастающих достаточно медленно.

Случай, при котором $F$ принадлежит области ненормального притяжения устойчивого закона ранее не рассматривался.

3. $\mathbf{E} X>0$. Условие (5) при дополнительных условиях на распределение $F$ проверяется так же, как в случаях 1 и 2 . Так как $\gamma(0)=\mathbf{E} X$, то (7) преврашается в

$$
\limsup \frac{W_{n}}{a_{n}}=\limsup \frac{U_{n}}{a_{n}}=\limsup \frac{R_{n}}{a_{n}}=\limsup \frac{T_{n}}{a_{n}}=\mathbf{E} X \quad \text { п.н. }
$$

Если $\ln \ln n=o\left(\ln \left(n / a_{n}\right)\right)$, то

$$
\lim \frac{W_{n}}{a_{n}}=\lim \frac{U_{n}}{a_{n}}=\mathbf{E} X \quad \text { п.н. }
$$

$\mathrm{C}$ помощью несложных рассуждений можно показать, что последние соотношения верны для $W_{n}$ и в случае $\mathbf{E} X=0$. Для остальных статистик эти соотношения верны и для $\mathbf{E} X<0$.

Отметим, что $R_{n}=S_{n}$ для $a_{n}=n$. В этом случае limsup можно заменить на $\mathrm{lim}$, так как доказательство достаточно провести для неотрицательных случайных величин, а для них $S_{n}$ не убывает. Таким образом, мы получили закон больших чисел.

Приведенные примеры показывают, что нормирующая последовательность во всех упоминавшихся выше сильных предельных теоремах для приращений сумм и сумм вычисляется по одной и той же формуле! Эта нормирующая последовательность зависит от поведения функции $\gamma(x)$. Поведение $\gamma(x)$ в окрестности нуля определяет асимптотику больших прирашений, ее поведение при ограниченных значениях аргумента - асимптотику приращений логарифмической длины, a eе поведение на бесконечности - асимптотику совсем коротких приращений. 
2. Доказательства. Нам понадобится следующая лемма. Положим $h_{0}^{\prime}=\infty$, если $F \in K_{\mathrm{V}}$, и $h_{0}^{\prime}=h_{0}$ в противоположном случае.

Лемма 1. Пусть выполненьи условия (A), (В) и (С). Тогда

$$
\mathbf{P}\left\{S_{n} \geqslant n m(h)\right\} \leqslant \exp \{-n f(h)\}
$$

для любого $h \in\left(0, h_{0}^{\prime}\right)$ u всех $n$.

Пусть $\left\{h_{n}\right\}$ - последовательность положительных чисел. Предположим, что выполнено также одно из следующих условий:

1) $h_{n}<h_{0}, n f\left(h_{n}\right) \rightarrow \infty u h_{n} \sigma\left(h_{n}\right)=o\left(\sqrt{n} f\left(h_{n}\right)\right)$;

2) $F \in K_{\text {III }}$ или $F \in K_{\mathrm{V}}, h_{n} \rightarrow \infty$ и неравенство (6) выполнено для любых $\tau>0, \delta>0$ и всех достаточно больших $h$;

3) $F \in K_{\mathrm{IV}}, h_{n} \nearrow h_{0}$ и неравенство (6) выполнено для любых $\tau>0$, $\delta>0$ и всех $h$ достаточно близких $\kappa h_{0}$;

4) $F \in K_{\mathrm{V}}, h_{n}=h_{*}>h_{0}$.

Тогда

$$
\mathbf{P}\left\{S_{n} \geqslant(1-\varepsilon) n m\left(h_{n}\right)\right\} \geqslant \exp \left\{-n f\left(h_{n}\right)(1+\delta)\right\}
$$

для любых $\varepsilon>0, \delta>0$ и всех достаточно больших $n$.

Д о к а $з$ а т е л ь с т в о. Для случая, когда справедливо условие 1) и $\mathbf{E} X=0$, оценки (16) и (17) были получены Феллером [13]. Заметим, что оценка сверху (16) следует из неравенства Чебышёва без выполнения условий 1) и $\mathbf{E} X=0$. Для $\mathbf{E} X>0$ оценка (17) вытекает из оценки для центрированной величины, неравенства $\mathbf{P}\left\{S_{n}+x \geqslant y+(1-\varepsilon) x\right\} \geqslant$ $\mathbf{P}\left\{S_{n} \geqslant y\right\}$, где $x>0$, и того факта, что при сдвиге случайной величины $X$ функция $f(h)$ не меняется, а $m(h)$ увеличивается на величину сдвига.

Если выполнено условие 2) или 3), то (17) следует из (6) и легко проверяемого неравенства $\mathbf{P}\left\{S_{n} \geqslant n x\right\} \geqslant(\mathbf{P}\{X \geqslant x\})^{n}$.

Пусть выполнено условие 4).

Сначала покажем, что для любых $\tau \in(0,1), \delta>0$ и $H>0$ существует $h>H$ такое, что

$$
\mathbf{P}\{h \geqslant X \geqslant(1-\tau) h\} \geqslant \exp \left\{-(1+\delta) h_{0} h\right\} .
$$

Предположим противное. Тогда найдутся $\tau \in(0,1), \delta>0$ и $H>0$ такие, что для любых $h>H$ выполнено неравенство противоположное (18). Обозначим $t_{k}=(1-\tau)^{-k}, k=1,2, \ldots$ Для всех достаточно больших $k$ справедливо неравенство

$$
\int_{(1-\tau) t_{k}}^{t_{k}} e^{h x} d F(x) \leqslant e^{h t_{k}} \mathbf{P}\left\{(1-\tau) t_{k} \leqslant X \leqslant t_{k}\right\}<e^{\left(h-(1+\delta) h_{0}\right) t_{k}} .
$$

Следовательно, $\varphi(h)<\infty$ для $0<h<(1+\delta) h_{0}$, что противоречит определению $h_{0}$. Неравенство (18) доказано. 
Пусть $\bar{X}, \bar{X}_{1}, \bar{X}_{2}, \ldots$ - последовательность независимых случайных величин с функцией распределения $\left(\varphi\left(h_{0}\right)\right)^{-1} \int_{-\infty}^{x} e^{h_{0} t} d F(t)$. Обозначим $\bar{S}_{n}=\bar{X}_{1}+\cdots+\bar{X}_{n}, \bar{F}_{n}(t)=\mathbf{P}\left\{\bar{S}_{n}<t\right\}$.

Пусть $\delta$ - фиксированное положительное число, $\tau=\delta / 2, \tau=6 \rho$.

Положим $z=h_{*}-h_{0}, x=n m\left(h_{*}\right), y=x+\tau n z, Q_{n}=\mathbf{P}\left\{x \leqslant \bar{S}_{n} \leqslant y\right\}$.

Используя известное соотношение, связываюшее функцию распределения сумм независимых случайных величин и функцию распределения сумм преобразований Крамера (см., например, [9]), получим

$$
P_{n}=\mathbf{P}\left\{S_{n} \geqslant x\right\}=\left(\varphi\left(h_{0}\right)\right)^{n} \int_{x}^{\infty} e^{-t h_{0}} d \bar{F}_{n}(t) \geqslant\left(\varphi\left(h_{0}\right)\right)^{n} e^{-h_{0} y} Q_{n}
$$

Пусть $a>1$ - фиксированное число, выбором которого распорядимся позднее. Положим $T_{n}=\bar{S}_{n}-\mathbf{E} \bar{S}_{n}, u=m\left(h_{0}\right)+(1+2 \rho) z a$, $v=m\left(h_{0}\right)+(1+3 \rho) z a$. Учитывая (3) и $\mathbf{E} \bar{S}_{n}=n m\left(h_{0}\right)$, имеем

$$
\begin{aligned}
Q_{n} & =\mathbf{P}\left\{z n \leqslant T_{n} \leqslant(1+6 \rho) z n\right\} \\
& \geqslant \mathbf{P}\left\{(1+2 \rho) z n \leqslant T_{n / a} \leqslant(1+3 \rho) z n\right\} \mathbf{P}\left\{-2 \rho z n \leqslant T_{n}-T_{n / a} \leqslant 3 \rho z n\right\} \\
& \geqslant \frac{1}{2} \mathbf{P}\left\{(1+2 \rho) z n \leqslant T_{n / a} \leqslant(1+3 \rho) z n\right\} \geqslant \frac{1}{2}(\mathbf{P}\{u \leqslant \bar{X} \leqslant v\})^{n / a}
\end{aligned}
$$

для всех достаточно больших $n$. Здесь мы использовали слабый закон больших чисел. Имеем

$$
Q_{n} \geqslant \frac{1}{2}\left(\frac{1}{\varphi\left(h_{0}\right)} \int_{u}^{v} e^{h_{0} t} d F(t)\right)^{n / a} \geqslant \frac{1}{2}\left(\frac{e^{h_{0} u}}{\varphi\left(h_{0}\right)} \mathbf{P}\{u \leqslant X \leqslant v\}\right)^{n / a}
$$

для всех достаточно больших $n$. Так как $\lim _{a \rightarrow \infty} u / v=\varepsilon \in(0,1)$, то, если $a$ было выбрано достаточно большим, выполняются неравенства

$$
\mathbf{P}\{u \leqslant X \leqslant v\} \geqslant \mathbf{P}\left\{v \varepsilon^{1 / 2} \leqslant X \leqslant v\right\} \geqslant \exp \left\{-\frac{1+4 \rho}{1+3 \rho} h_{0} v\right\} .
$$

В последнем неравенстве мы использовали (18). Из (19)-(21) следует, что для всех достаточно больших $n$ имеет место неравенство $P_{n} \geqslant$ $\frac{1}{2} \exp \{n p(a)\}$, где $p(a)$ не зависит от $n$ и $\lim _{a \rightarrow \infty} p(a)=-f\left(h_{*}\right)-8 \rho h_{0} z \geqslant$ $-(1+8 \rho) f\left(h_{*}\right)$. Если $a$ было выбрано достаточно большим, то отсюда мы получим (17).

Мы будем использовать следуюшую известную лемму.

Лемма 2. Пусть $r, s \geqslant 0, q>0$. Если $\mathbf{P}\left\{S_{i} \geqslant-s\right\} \geqslant q$ для всех $i=1,2, \ldots, n, m o$

$$
\mathbf{P}\left\{\max _{0 \leqslant i<j \leqslant n}\left(S_{j}-S_{i}\right) \geqslant r\right\} \leqslant q^{-2} \mathbf{P}\left\{S_{n} \geqslant r-s\right\} .
$$

В доказательстве теоремы 1 ключевое место занимает следующая лемма. 
Лемма 3. Пусть $A_{n}(\varepsilon)=\left\{W_{n} \geqslant(1+\varepsilon) b_{n}\right\}$, где $\varepsilon>0$. Если выполнень условия теоремь 1 , то для любого $\varepsilon>0$ существует $\tau>0$ такое, что для всех достаточно больших $п$ выполняется неравенство

$$
\mathbf{P}\left(A_{n}(\varepsilon)\right) \leqslant(\ln n)^{-(1+\tau)} .
$$

Д ок аз а т ел ь с т в. Положим

$$
\beta_{n}=\ln \frac{n}{a_{n}}+\ln \ln n .
$$

Рассмотрим сначала случай $\ln a_{n} / \ln n \rightarrow 0$. Используя выпуклость вверх функции $\gamma(x)$, получим $(1+\varepsilon) b_{n} \geqslant k \gamma\left((1+\varepsilon) \beta_{n} / k\right)$ при $1 \leqslant k \leqslant a_{n}$. С учетом (16) и (2) имеем

$$
\begin{aligned}
\mathbf{P}\left(A_{n}(\varepsilon)\right) & \leqslant n \sum_{k=1}^{a_{n}} \mathbf{P}\left\{S_{k} \geqslant(1+\varepsilon) b_{n}\right\} \leqslant n \sum_{k=1}^{a_{n}} \mathbf{P}\left\{S_{k} \geqslant k \gamma\left((1+\varepsilon) \frac{\beta_{n}}{k}\right)\right\} \\
& \leqslant n \sum_{k=1}^{a_{n}} \exp \left\{-(1+\varepsilon) \beta_{n}\right\}=n^{-\varepsilon} a_{n}^{2+\varepsilon}(\ln n)^{-(1+\varepsilon)} .
\end{aligned}
$$

Отсюда следует (22). Отметим, что здесь возможен случай $a_{n}=$ const .

Перейдем ко второму случаю. Пусть $a_{n} \rightarrow \infty, a_{n} / n<1-\delta$ при всех достаточно больших $n$, где $\delta \in(0,1)$. Используя лемму 2 и условие 2$)$, имеем

$$
\begin{aligned}
& \mathbf{P}\left(A_{n}(2 \varepsilon)\right) \\
& \quad \leqslant \sum_{j=1}^{\left[\left(n-a_{n}\right) /\left(\varepsilon a_{n}\right)\right]-1} \mathbf{P}\left\{\max _{(j-1) \varepsilon a_{n} \leqslant m \leqslant j \varepsilon a_{n}} \max _{1 \leqslant k \leqslant a_{n}}\left(S_{k+m}-S_{m}\right) \geqslant(1+2 \varepsilon) b_{n}\right\} \\
& \quad \leqslant \frac{n}{\varepsilon a_{n}} \mathbf{P}\left\{\max _{0 \leqslant m \leqslant \varepsilon a_{n}} \max _{1 \leqslant k \leqslant a_{n}}\left(S_{k+m}-S_{m}\right) \geqslant(1+2 \varepsilon) b_{n}\right\} \\
& \quad \leqslant \frac{n}{\varepsilon a_{n} q^{2}} \mathbf{P}\left\{S_{(1+\varepsilon) a_{n}} \geqslant(1+\varepsilon) b_{n}\right\} \\
& \quad=\frac{n}{\varepsilon a_{n} q^{2}} \mathbf{P}\left\{S_{(1+\varepsilon) a_{n}} \geqslant(1+\varepsilon) a_{n} \gamma\left(\frac{\beta_{n}}{a_{n}}\right)\right\}
\end{aligned}
$$

для всех достаточно больших $n$. Отсюда и из соотношений (16) и (2) следует (22).

Осталось рассмотреть последний случай $a_{n} / n \rightarrow 1$. Положим $W_{n}^{\prime}=$ $\max _{0 \leqslant k<j \leqslant n}\left(S_{j}-S_{k}\right)$. Тогда

$$
\mathbf{P}\left(A_{n}(2 \varepsilon)\right) \leqslant \mathbf{P}\left\{W_{n}^{\prime} \geqslant(1+2 \varepsilon) b_{n}\right\} \leqslant \mathbf{P}\left\{W_{(1+\varepsilon) n}^{\prime} \geqslant(1+2 \varepsilon) b_{n}\right\} .
$$

Снова применяя лемму 2 , условие 2 ), соотношения (16) и (2), приходим к (22).

Доказательство те оремы 1. Положим $n_{k}=\left[\theta^{k}\right]$, $\theta \in\left(1, \theta_{0}\right)$. Из соотношения $(22)$ и леммы Бореля-Кантелли следует, что

$$
\limsup _{k \rightarrow \infty} \frac{W_{n_{k}}}{b_{n_{k}}} \leqslant 1 \quad \text { п.н. }
$$


Далее, $b_{n_{k}} \leqslant b_{n_{k+1}} \leqslant b_{n_{k}} g(\theta)$ для всех достаточно больших $k$. Так как $W_{n}$ не убывает, то $W_{n} / b_{n} \leqslant g(\theta) W_{n_{k+1}} / b_{n_{k+1}}$ при $n_{k} \leqslant n \leqslant n_{k+1}$. Из последних неравенств и (24) следует, что $\lim \sup W_{n} / b_{n} \leqslant g(\theta)$ п.н. Устремляя в последнем соотношении $\theta$ к 1 , мы получаем требуемое.

Лемма 4. Пусть $A_{n}^{\varepsilon}=\left\{T_{n} \geqslant(1-\varepsilon) b_{n}\right\}$, где $\varepsilon>0$. Если выполнень условия теоремы 2 , то для любого $\varepsilon>0$ существует $\tau>0$ такое, что для всех достаточно больших $n$ выполняется неравенство

$$
\mathbf{P}\left(A_{n}^{\varepsilon}\right) \geqslant \exp \left\{-(1-\tau)\left(\ln \frac{n}{a_{n}}+\ln \ln n\right)\right\} .
$$

Д о к а з а т е л ь с т в о. Определим $\beta_{n}$ равенством (23).

Пусть выполнено одно из условий 1), 4)-6) теоремы 2 . В силу выпуклости вверх функции $\gamma(x)$ выполняется неравенство $(1-\varepsilon)^{2} b_{n} \leqslant$ $(1-\varepsilon) a_{n} \gamma\left((1-\varepsilon) \beta_{n} / a_{n}\right)$. Используя (17) и (2), имеем

$$
\begin{aligned}
\mathbf{P}\left(A_{n}^{2 \varepsilon-\varepsilon^{2}}\right) & =\mathbf{P}\left\{S_{a_{n}} \geqslant(1-\varepsilon)^{2} b_{n}\right\} \\
& \geqslant \mathbf{P}\left\{S_{a_{n}} \geqslant(1-\varepsilon) \gamma\left((1-\varepsilon) \beta_{n} a_{n}^{-1}\right)\right\} \geqslant \exp \left\{-(1+\delta)(1-\varepsilon) \beta_{n}\right\}
\end{aligned}
$$

для всех достаточно больших $n$. Выбирая $\delta$ достаточно малым, приходим к (25).

Если выполнено условие 2) теоремы 2 , то $b_{n}=a_{n} \omega, \beta_{n} / a_{n} \rightarrow \infty$, и $\mathbf{P}\left(A_{n}^{\varepsilon}\right) \geqslant(\mathbf{P}\{X \geqslant(1-\varepsilon) \omega\})^{a_{n}} \geqslant \exp \left\{-(1-\tau) \beta_{n}\right\}$ для всех достаточно больших $n$.

Если выполнено условие 3) теоремы 2 , то снова $b_{n}=a_{n} \omega$. Далее считаем, что $a_{n} \rightarrow \infty$, так как в противном случае (25) выполнено автоматически. Пусть $m$ - натуральное число, выбором которого распорядимся позднее. Имеем

$$
\mathbf{P}\left(A_{n}^{\varepsilon}\right) \geqslant\left(\mathbf{P}\left\{S_{m} \geqslant(1-\varepsilon) \omega m\right\}\right)^{a_{n} / m} \geqslant\left(\mathbf{P}\left\{S_{m} \geqslant(1-\varepsilon) \omega m\right\}\right)^{c_{0} \beta_{n} / m} .
$$

Возьмем $x_{0}$ такое, что $F\left(x_{0}\right)<F(\omega)$. Выберем $m$ столь большим, чтобы $(1-\varepsilon) m \omega \leqslant x_{0}+(m-1) \omega$. Тогда

$$
\begin{aligned}
& \mathbf{P}\left\{S_{m} \geqslant(1-\varepsilon) \omega m\right\} \geqslant \mathbf{P}\left\{S_{m} \geqslant x_{0}+(m-1) \omega\right\} \\
& \quad \geqslant m \mathbf{P}\left\{\omega>X_{1} \geqslant x_{0}, X_{2}=\cdots=X_{m}=\omega\right\} \\
& \quad=m \mathbf{P}\left\{\omega>X \geqslant x_{0}\right\}(\mathbf{P}\{X=\omega\})^{m-1}=m\left(F(\omega)-F\left(x_{0}\right)\right) e^{-(m-1) / c_{0}} .
\end{aligned}
$$

Отсюда мы получаем (25).

Док а зат ел в с т в о т е о ре мы 2. По лемме 4

$$
\begin{aligned}
P_{n} & =\mathbf{P}\left\{R_{n} \geqslant(1-\varepsilon) b_{n}\right\}=\mathbf{P}\left(A_{n}^{\varepsilon}\right) \\
& \geqslant \exp \left\{-(1-\delta)\left(\ln \frac{n}{a_{n}}+\ln \ln n\right)\right\}
\end{aligned}
$$

для всех достаточно больших $n$. 
Пусть сначала $a_{n} / n \rightarrow \rho \in[0,1)$. Выберем $\tau \in(\rho, 1)$ и натуральное $N$ такими, что для всех $n>N$ выполняются неравенства $\left(a_{n}+1\right) / n<\tau$ и (26). Положим $n_{1}=N, n_{k+1}=\min \left\{n: n>n_{k}, n-a_{n} \geqslant\right.$ $\left.n_{k}\right\}, k=2,3, \ldots$ Используя неравенство $a_{n} / n \geqslant\left(a_{n}+1\right) /(2 n)$, получим

$$
\sum_{k=2}^{m} P_{n_{k}} \geqslant \frac{1}{2}\left(\ln n_{m}\right)^{-1+\delta} \sum_{k=2}^{m} \frac{a_{n_{k}}+1}{n_{k}} .
$$

В силу неравенств $n_{k}-a_{n_{k}}-1 \leqslant n_{k}-a_{n_{k}-1}-1<n_{k-1}$ и $-\ln (1-x) \leqslant C_{\tau} x$ при $x \in(0, \tau)$, имеем

$$
C_{\tau} \sum_{k=2}^{m} \frac{a_{n_{k}}+1}{n_{k}} \geqslant \sum_{k=2}^{m} \ln \frac{n_{k}}{n_{k}-a_{n_{k}}-1} \geqslant \sum_{k=2}^{m} \ln \frac{n_{k}}{n_{k-1}}=\ln \frac{n_{m}}{n_{1}} .
$$

Следовательно, ряд $\sum_{k} P_{n_{k}}$ расходится. Так как события $\left\{R_{n_{k}} \geqslant\right.$ $\left.(1-\varepsilon) b_{n_{k}}\right\}$ независимы, то заключение теоремы следует из леммы Бореля-Кантелли.

Пусть теперь $a_{n} / n \rightarrow 1$. Положим $n_{k}=\left[\theta^{k}\right], k=1,2, \ldots, \theta>1$. Покажем, что соотношение

$$
\limsup _{k \rightarrow \infty} \frac{R_{n_{k}}}{b_{n_{k}}} \leqslant a<1 \quad \text { п.н. }
$$

не выполнено.

Рассмотрим события $A_{k}=\left\{S_{n_{k}}-S_{n_{k-1}}>(1+2 \varepsilon) a b_{n_{k}}\right\}, D_{k}=$ $\left\{S_{n_{k-1}}-S_{n_{k}-a_{n_{k}}}>-\varepsilon a b_{n_{k}}\right\}, k=1,2, \ldots$

Так как $n_{k-1}-n_{k}+a_{n_{k}} \sim n_{k-1}$, то $n_{k-1}-n_{k}+a_{n_{k}} \leqslant n_{k}$ для всех достаточно больших $k$. Из условия 2) следует, что $\mathbf{P}\left\{D_{k}\right) \geqslant q$ для всех достаточно больших $k$.

Следующие пары событий независимы: $A_{k}$ и $D_{k}, A_{k}$ и $D_{k} \overline{A_{k-1} D_{k-1}}$, $A_{k}$ и $D_{k} \overline{A_{k-1} D_{k-1} A_{k-2} D_{k-2}}, \ldots$

Так как $a_{n_{k}} \sim\left(n_{k}-n_{k-1}\right) \theta /(\theta-1)$, то

$$
\mathbf{P}\left(A_{k}\right) \geqslant \mathbf{P}\left\{S_{n_{k}-n_{k-1}}>(1+\varepsilon) a \frac{\theta}{\theta-1}\left(n_{k}-n_{k-1}\right) \gamma\left(\frac{\beta_{n_{k}}}{a_{n_{k}}}\right)\right\}
$$

для всех достаточно больших $k$. Выберем $\theta$ большим, а $\varepsilon$ маленьким так, чтобы $(1+\varepsilon) a \theta /(\theta-1)=(1-\tau)^{2}, \tau>0$. Используя выпуклость $\gamma(x)$ и соотношения (17) и (2) так же, как при доказательстве леммы 4 , получим

$$
\mathbf{P}\left(A_{k}\right) \geqslant \exp \left\{-(1-\tau)(1+\delta)\left(n_{k}-n_{k-1}\right) \beta_{n_{k}} a_{n_{k}}^{-1}\right\} \geqslant k^{-(1-\tau)(1+\delta)}
$$

для всех достаточно больших $k$. Выбирая $\delta$ достаточно малым, заключаем, что ряд $\sum_{k} \mathbf{P}\left(A_{k}\right)$ расходится.

По лемме 5 на с. 267 книги Петрова [11], $\mathbf{P}\left(A_{k} D_{k}\right.$ б.ч. $) \geqslant q>0$. $\mathrm{C}$ другой стороны, из $(27)$ следует, что $\mathbf{P}\left(A_{k} D_{k}\right.$ б.ч. $)=0$. Полученное противоречие показывает, что (27) не выполнено, и теорема доказана для $R_{n}$. 
Доказательство для $T_{n}$ в силу $(25)$ не отличается от доказательства леммы 4 в работе автора [8] и здесь опущено.

Д оказател ь с т о т е о е мы 3. Доказательство в силу неравенства (25) не отличается от доказательства леммы 5 в работе автора [8] и здесь опушено.

Перейдем к доказательству утверждений, сделанных ранее по поводу проверки условий теоремы 4 в случае III.

Докажем сначала соотношения (9)-(12), (14), (15).

Пусть $\mathbf{E} X=0, \sigma^{2}=\mathbf{E} X^{2}<\infty$. Покажем, что выполнены (9) и (10). По формуле Тейлора

$$
\varphi(h)=1+\frac{h^{2}}{2} \varphi^{\prime \prime}\left(\theta_{1}\right), \quad \ln \varphi(h)=\frac{h^{2}}{2} \sigma^{2}\left(\theta_{2}\right), \quad m(h)=h \sigma^{2}\left(\theta_{3}\right),
$$

где $\theta_{i}=\theta_{i}(h), \theta_{i} \in(0, h), i=1,2,3$. Следовательно,

$$
f(h)=h m(h)-\ln \varphi(h)=\frac{h^{2}}{2}\left(2 \sigma^{2}\left(\theta_{3}\right)-\sigma^{2}\left(\theta_{2}\right)\right) .
$$

Переходя во всех этих соотношениях к пределу при $h \rightarrow 0$ и учитывая соотношения $\varphi^{\prime \prime}(h) \rightarrow \sigma^{2}$ и $\sigma^{2}(h) \rightarrow \sigma^{2}$ при $h \rightarrow 0$, получим (9) и (10).

Пусть $\mathbf{E} X=0, \mathbf{E} X^{2}=\infty$ и $F$ принадлежит области притяжения нормального закона. Покажем, что выполнены (11) и (12).

Положим $R_{0}(x)=e^{x}-1-x, R_{1}(x)=x\left(e^{x}-1\right), R_{2}(x)=x^{2} e^{x}$,

$$
I_{k}=h^{-k} \int_{-\infty}^{\infty} R_{k}(x h) d F(x), \quad k=0,1,2 .
$$

Тогда

$$
\begin{gathered}
\varphi(h)=1+I_{0}, \\
\varphi(h) m(h)=I_{1}, \\
\left(\sigma^{2}(h)+(m(h))^{2}\right) \varphi(h)=I_{2} .
\end{gathered}
$$

Исследуем поведение интегралов $I_{k}$ при $h \rightarrow 0$. Имеем

$$
\begin{aligned}
I_{k} & =h^{-k}\left(\int_{-\infty}^{-1 / h}+\int_{-1 / h}^{0}+\int_{0}^{\infty}\right) R_{k}(x h) d F(x) \\
& =I_{k}^{\prime}+I_{k}^{\prime \prime}+I_{k}^{\prime \prime \prime}, \quad k=0,1,2 .
\end{aligned}
$$

Так как $R_{k}(x) \leqslant x^{2}((2-k) !)^{-1} e^{x} \leqslant x^{2} e^{x}, x>0$, то для $k=0,1,2$

$$
I_{k}^{\prime \prime \prime}=O\left(h^{2-k}\right) \quad \text { при } \quad h \rightarrow 0 .
$$

Так как $F$ принадлежит области притяжения нормального закона, a $1-F(x)$ убывает при $x \rightarrow \infty$ экспоненциально (в силу условия $(\mathrm{C})$ ), то

$$
F(-x)=x^{-2} g(x) \quad \text { при } \quad x>0,
$$


где $g(x)$ - медленно меняющаяся функция (см., например, [15, с. 97]). Интегрируя по частям и делая замену переменных $h x \rightarrow-x$, получим

$$
\begin{aligned}
I_{k}^{\prime} & =h^{-k} R_{k}(-1) F\left(-\frac{1}{h}\right)-h^{-k} \int_{1}^{\infty} F\left(-\frac{x}{h}\right) R_{k}^{\prime}(-x) d x \\
& =h^{2-k} R_{k}(-1) g\left(\frac{1}{h}\right)-h^{2-k} \int_{1}^{\infty} g\left(\frac{x}{h}\right) x^{-2} R_{k}^{\prime}(-x) d x .
\end{aligned}
$$

По теореме 2.6 на с. 63 книги [14] для $k=0,1,2$

$-\int_{1}^{\infty} g\left(\frac{x}{h}\right) x^{-2} R_{k}^{\prime}(-x) d x \sim-g\left(\frac{1}{h}\right) \int_{1}^{\infty} x^{-2} R_{k}^{\prime}(-x) d x \quad$ при $\quad h \rightarrow 0$.

Поэтому для $k=0,1,2$

$$
I_{k}^{\prime} \sim C_{k} h^{2-k} g\left(h^{-1}\right) \quad \text { при } \quad h \rightarrow 0 .
$$

Положим $L_{k}(x)=R_{k}-x^{2} /(2-k) !, k=0,1,2$. Снова интегрируя по частям, делая замену переменных $h x \rightarrow-x$ и учитывая (32), получим

$$
\begin{aligned}
I_{k}^{\prime \prime} & =h^{-k} \int_{-1 / h}^{0} L_{k}(x h) d F(x)+\frac{h^{2-k}}{(2-k) !} \int_{-1 / h}^{0} x^{2} d F(x) \\
& =-h^{2-k} L_{k}(-1) g\left(\frac{1}{h}\right)-h^{2-k} \int_{0}^{1} g\left(\frac{x}{h}\right) x^{-2} L_{k}^{\prime}(-x) d x+\frac{h^{2-k}}{(2-k) !} G\left(\frac{1}{h}\right) .
\end{aligned}
$$

По теореме 2.7 на с. 65 книги [14] для $k=0,1,2$

$$
\int_{0}^{1} g\left(\frac{x}{h}\right) x^{-2} L_{k}^{\prime}(-x) d x \sim g\left(\frac{1}{h}\right) \int_{0}^{1} x^{-2} L_{k}^{\prime}(-x) d x \quad \text { при } \quad h \rightarrow 0 .
$$

В силу условия $(\mathrm{C})$ имеем $G(y) \sim G_{1}(y)=\int_{-y}^{y} x^{2} d F(x)$ и $g(y) \sim$ $g_{1}(y)=1-F(y)+F(-y)$ при $y \rightarrow \infty$. Хорошо известно (см., например, $\left[15\right.$, c. 101]), что $g_{1}(y)=o\left(G_{1}(y)\right)$ при $y \rightarrow \infty$. Поэтому для $k=0,1,2$

$$
I_{k}^{\prime \prime} \sim \frac{h^{2-k}}{(2-k) !} G\left(\frac{1}{h}\right) \quad \text { при } \quad h \rightarrow 0 .
$$

Из (31), (34), (35) вытекает, что для $k=0,1,2$

$$
I_{k} \sim \frac{h^{2-k}}{(2-k) !} G\left(\frac{1}{h}\right) \quad \text { при } \quad h \rightarrow 0 .
$$

Из (28) и (36) для $k=0$ вытекает

$$
\varphi(h)=1+\frac{h^{2}}{2} G\left(\frac{1}{h}\right)(1+o(1)) \quad \text { при } \quad h \rightarrow 0 .
$$

Из последнего соотношения, (29) и (36) для $k=1$ следует, что

$$
m(h)=h G\left(h^{-1}\right)(1+o(1)) \quad \text { при } \quad h \rightarrow 0 .
$$

Из последнего соотношения, (37), (30) и (36) для $k=2$ следует, что

$$
\sigma^{2}(h)=G\left(h^{-1}\right)(1+o(1)) \quad \text { при } \quad h \rightarrow 0 .
$$


Из (37) и (38) заключаем, что

$$
f(h)=\frac{h^{2}}{2} G\left(\frac{1}{h}\right)(1+o(1)) \quad \text { при } \quad h \rightarrow 0 .
$$

Осталось только заметить, что (37)-(40) совпадают с (11)-(12).

Пусть $\mathbf{E} X=0, \mathbf{E} X^{2}=\infty$ и $F$ принадлежит области притяжения устойчивого закона с показателем $\alpha$. Покажем, что выполнены (14) и (15).

Доказательство мало отличается от доказательства в случае притяжения к нормальному закону. Определим $R_{k}$ и $I_{k}$ так же, как раньше. Представим $\varphi(h), m(h), \sigma^{2}(h)$ в виде $(28)-(30)$ и исследуем поведение интегралов $I_{k}$ при $h \rightarrow 0$.

Имеем

$$
\begin{aligned}
I_{k} & =h^{-k} \int_{-\infty}^{0} R_{k}(x h) d F(x)+h^{-k} \int_{0}^{\infty} R_{k}(x h) d F(x) \\
& =J_{k}^{\prime}+J_{k}^{\prime \prime}, \quad k=0,1,2 .
\end{aligned}
$$

Интеграл $J_{k}^{\prime \prime}$ оценивается так же, как $I_{k}^{\prime \prime \prime}$ : для $k=0,1,2$

$$
J_{k}^{\prime \prime}=O\left(h^{2-k}\right) \quad \text { при } \quad h \rightarrow 0 .
$$

Так как $F$ принадлежит области притяжения устойчивого закона с показателем $\alpha$, а $1-F(x)$ убывает при $x \rightarrow \infty$ экспоненциально (в силу условия (C)), то $F(-x)=x^{-\alpha} g(x)$, где $g(x)$ - медленно меняюшаяся функция (см., например, [15, с. 93]). Интегрируя по частям и делая замену переменных $h x \rightarrow-x$, получим

$J_{k}^{\prime}=-h^{-k} \int_{0}^{\infty} F\left(-\frac{x}{h}\right) R_{k}^{\prime}(-x) d x=-h^{\alpha-k} \int_{0}^{\infty} g\left(-\frac{x}{h}\right) x^{-\alpha} R_{k}^{\prime}(-x) d x$.

Дальнейшие рассуждения ничем не отличаются от рассуждений, использованных при доказательстве соотношения (36). Нужно лишь заменить 2 на $\alpha$ во всех интегралах. Тогда мы получим

$$
I_{k} \sim C_{k} h^{\alpha-k} g\left(\frac{1}{h}\right) \quad \text { при } \quad h \rightarrow 0 \quad(k=0,1,2) .
$$

Из последнего соотношения и (28)-(30) мы тем же способом получим (14) и (15).

Перейдем к проверке условий 2) и (5).

Начнем с условия (5). Заметим, что во всех рассматриваемых случаях, где $\mathbf{E} X=0$ и $a_{n} / \ln n \rightarrow \infty$, выполняются соотношения $h^{*} \rightarrow 0$ и $h^{2} \sigma^{2}(h) \sim C f(h)$ при $h \rightarrow 0$. Поэтому условие (5) равносильно соотношению $\left(h^{*}\right)^{2} \sigma^{2}\left(h^{*}\right)=o\left(a_{n}\left(f\left(h^{*}\right)\right)^{2}\right)$, которое, в свою очередь, эквивалентно $1=o\left(a_{n} f\left(h^{*}\right)\right)=o\left(\beta_{n}\right)$. Последнее соотношение выполнено, так как $a_{n} / \ln n \rightarrow \infty$.

Перейдем к условию 2). Для его проверки полезна следующая лемма. 
Лемма 5. Пусть выполнено условие (В). Пусть $\left\{B_{n}\right\}-$ неубывающая последовательность положительных постоянных такая, что функиии распределения нормированных сумм $S_{n} / B_{n}$ слабо сходятся $\kappa$ функиии распределения $V(x)$. Тогда для любого $\delta>0$ такого, что $-\delta$ - точка непрерывности $V(x)$, существует $q>0$ такое, что для всех $i \leqslant n$ и всех $n$ выполняется неравенство $\mathbf{P}\left\{S_{i} \geqslant-\delta B_{n}\right\} \geqslant q$.

Д ок аз а т ел с с т в . В силу условия леммы $\mathbf{P}\left\{S_{i} \geqslant-\delta B_{i}\right\} \geqslant$ $(1-V(-\delta)) / 2$ для всех $i \geqslant I_{0}=I_{0}(\delta)$. Для $i<I_{0}$ имеем

$$
\mathbf{P}\left\{S_{i} \geqslant-\delta B_{i}\right\} \geqslant \mathbf{P}\left\{S_{i} \geqslant 0\right\} \geqslant \mathbf{P}\left\{X_{1} \geqslant 0, \ldots, X_{i} \geqslant 0\right\} \geqslant(\mathbf{P}\{X \geqslant 0\})^{I_{0}}>0,
$$

так как $\mathbf{E} X \geqslant 0$. Значит для всех $i$ выполняется неравенство

$$
\mathbf{P}\left\{S_{i} \geqslant-\delta B_{i}\right\} \geqslant q=\min \left\{2^{-1}(1-V(-\delta)),(\mathbf{P}\{X \geqslant 0\})^{I_{0}}\right)>0 .
$$

Так как $B_{n}$ не убывает, то $\mathbf{P}\left\{S_{i} \geqslant-\delta B_{n}\right\} \geqslant \mathbf{P}\left\{S_{i} \geqslant-\delta B_{i}\right\} \geqslant q$ для всех $i \leqslant n$.

Теперь покажем, что условие 2) выполнено.

1. $\mathbf{E} X=0, \sigma^{2}=\mathbf{E} X^{2}<\infty$. Пусть $a_{n} / n<1-\delta$ для всех достаточно больших $n$. Положим $\kappa_{n}=\left(\left[(1+\varepsilon) a_{n}\right] \sigma^{2} /(1-q)\right)^{1 / 2}$. По неравенству Чебышёва $\mathbf{P}\left\{S_{i} \geqslant-\kappa_{n}\right\} \geqslant 1-i \sigma^{2} \kappa_{n}^{-2} \geqslant q$ для $i \leqslant\left[(1+\varepsilon) a_{n}\right]$. Так как $\kappa_{n}=o\left(b_{n}\right)$, то мы получаем (4). Случай $a_{n} / n \rightarrow 1$ рассматривается аналогично.

2. $\mathbf{E} X=0, \mathbf{E} X^{2}=\infty$.

a) Возьмем $B_{n}=1 / f^{-1}(1 /(2 n))$. Тогда в силу (12) имеем $1=$ $2 n f\left(1 / B_{n}\right) \sim n B_{n}^{-2} G\left(B_{n}\right)$. Поэтому распределения $S_{n} / B_{n}$ сходятся к нормальному закону.

Положим $\kappa_{n}=B_{a_{n}}$. Покажем, что $\kappa_{n}=o\left(b_{n}\right)$. Определим $\beta_{n}$ равенством (23). Так как $m(h) \sim C f(h) / h$ при $h \rightarrow 0$, то

$$
\frac{\kappa_{n}}{b_{n}}=\frac{\kappa_{n}}{a_{n} m\left(f^{-1}\left(\beta_{n} / a_{n}\right)\right)} \sim \frac{\kappa_{n} f^{-1}\left(\beta_{n} / a_{n}\right)}{C \beta_{n}}=\frac{f^{-1}\left(\beta_{n} / a_{n}\right)}{C f^{-1}\left(1 /\left(2 a_{n}\right)\right) \beta_{n}} .
$$

Нам потребуется представление произвольной медленно меняющейся функции $L(x)$ в виде

$$
L(x)=D(1+o(1)) \exp \left\{\int_{A}^{x} \frac{\varepsilon(t)}{t} d t\right\},
$$

где $\varepsilon(t) \rightarrow 0, t \rightarrow \infty$ (см., например, [12, теорема 1.2 , с. 10]).

Используя это представление и (12), нетрудно проверить, что $f^{-1}(h) \sim h^{1 / 2} L(1 / h)$ при $h \rightarrow 0$, где $L(x)$ - медленно меняющаяся функция. Поэтому $\kappa_{n} / b_{n} \sim L\left(a_{n} / \beta_{n}\right) /\left(C_{1} L\left(a_{n}\right) \beta_{n}^{1 / 2}\right)$. Используя снова представление медленно меняющейся функции, заключаем, что для любого $\delta>0$ и всех достаточно больших $n$ выполняется неравенство $L\left(a_{n} / \beta_{n}\right) \leqslant \beta_{n}^{\delta} L\left(a_{n}\right)$. Выбирая $\delta<\frac{1}{2}$, получаем $\kappa_{n}=o\left(b_{n}\right)$.

Теперь неравенство (4) следует из леммы 5. 
b) Так как $F$ устойчива, то для $i \leqslant\left[(1+\varepsilon) a_{n}\right]$ и $\kappa_{n}=\left(\left[(1+\varepsilon) a_{n}\right]\right)^{1 / \alpha}$ выполняется неравенство $\mathbf{P}\left\{S_{i} \geqslant-\kappa_{n}\right\}=\mathbf{P}\left\{i^{-1 / \alpha} S_{i} \geqslant-i^{-1 / \alpha} \kappa_{n}\right\} \geqslant$ $F(-1)>0$. Кроме того, ясно, что $\kappa_{n}=o\left(b_{n}\right)$. Это дает неравенство (4) для $\left\{a_{n}\right\}$ таких, что $a_{n} / n<1-\delta$ для всех достаточно больших $n$. Случай $a_{n} / n \rightarrow 1$ рассматривается аналогично.

c) Возьмем $B_{n}=1 / f^{-1}\left(c_{4} / n\right)$. Тогда в силу (15) имеем $1=$ $n f\left(1 / B_{n}\right) / c_{4} \sim n B_{n}^{-\alpha} g\left(B_{n}\right)$. Поэтому распределения $S_{n} / B_{n}$ слабо сходятся к соответствующему устойчивому закону. Положим $\kappa_{n}=B_{a_{n}}$. Соотношение $\kappa_{n}=o\left(b_{n}\right)$ доказывается так же, как в случае а). Остается применить лемму 5 .

3. $\mathbf{E} X>0$. Условие 2) выполнено в силу слабого закона больших чисел и леммы 5 .

Автор благодарен рецензенту за ряд ценных замечаний, способствовавших улучшению текста статьи.

\section{СПИСОК ЛИТЕРАТУРЫ}

1. Shepp L. A. A limit law concerning moving averages. - Ann. Math. Statist., 1964, v. 35 , p. $424-428$.

2. Erdös P., Rényi A. On a new law of large numbers. - J. Anal. Math., 1970, v. 23, p. $103-111$.

3. Csörgö S. Erdős-Rényi laws. - Ann. Statist., 1979, v. 7, p. 772-787.

4. Deheuvels P., Devroye L. Limit laws of Erdős-Rényi-Shepp type. - Ann. Probab., 1987 , v. 15 , p. $1363-1386$.

5. Mason D. M. An extended version of the Erdös-Rényi strong law of large numbers. Ann. Probab., 1989, v. 17, p. 257-265.

6. Csörgő M., Révész P. Strong Approximations in Probability and Statistics. Budapest: Akadémiai Kiadó, 1981, 285 p.

7. Зинченко Н. М. Асимптотика прирашений устойчивых случайных процессов со скачками одного знака. - Теория вероятн. и ее примен., 1987, т. 32 , в. 4, с. 793 796.

8. Frolov A. N. On one-sided strong laws for large increments of sums. - Statist. Probab. Lett., 1998, v. 37, № 2, p. 155-165.

9. Петров В.В. О вероятностях больших уклонений сумм независимых случайных величин. - Теория вероятн. и ее примен., 1965, т. 10, в. 2, с. 310-322.

10. Боровков A. A. Теория вероятностей. М.: Наука, 1986, 432 с.

11. Петров В. В. Предельные теоремы для сумм независимых случайных величин. М.: Наука, 1987,320 c.

12. Золотарев В. М. Одномерные устойчивые распределения. М.: Наука, 1983, 304 с.

13. Feller $W$. Limit theorems for probabilities of large deviations. - Z Wahrscheinlichkeitstheor. verw. Geb., 1969, v. 14, p. 1-20.

14. Сенета Е. Правильно меняющиеся функции. М.: Наука, 1985, 144 с.

15. Ибрагимов И.А., Линник Ю.В. Независимые и стационарно связанные величины. М.: Наука, 1965, 524 с. 